\title{
Roflumilast succeeds where traditional therapy fails: a case report
}

\author{
Gabriella Guarnieri1*
}

ABSTRACT: Roflumilast succeeds where traditional therapy fails: a case report.

The case of a 72-year-old man with a long history of chronic obstructive pulmonary disease (COPD, patient D according to Guidelines GOLD 2013) in a subject professionally exposed to welding fumes is presented. Diagnosis was based on symptoms and spirometry and confirmed by chest X-ray examination. Since 1997 the patient has been under different therapies, including high-dose inhaled corticosteroids and bronchodilators, with poor clinical control and frequent exacerbations. Roflumilast $500 \mu \mathrm{g}$ once daily was started in January 2012 and patient's respiratory symptoms, number of exacerbations and spirometry values have gradually improved since then. Roflumilast was an effective treatment in this case of difficult to treat severe COPD.

Monaldi Arch Chest Dis 2013; 79: 3-4, Suppl., 29-34.

Keywords: Roflumilast, COPD, Case report.

1 Occupational Medicine, Respiratory Pathophysiology Laboratory; Department of Cardiologic, Thoracic and Vascular Sciences, University of Padova, Padova, Italy.

Correspondence: Gabriella Guarnieri, MD, Occupational Medicine, Respiratory Pathophysiology Laboratory; Department of Cardiologic, Thoracic and Vascular Sciences, University of Padova, Via Giustiniani 2, 35128 Padova, Italy; e-mail: gabriella.guarnieri@unipd.it

\section{Case Report}

A 57-year-old man presented to our Department in 1997 when he was hospitalized for progressively worsening exertional dyspnea (modified medical research council mMRC - scale grade 1) and productive cough, which he had had since 1990 . The patient was a former smoker (16.5 pack-years), and at the time of first visit had been work- ing for 20 years as a welder in a metalworking company. He had no further co-morbidities. Post bronchodilator (BD) spirometry showed a forced expiratory volume in the first second $\left(\mathrm{FEV}_{1}\right)$ of $2.25 \mathrm{~L}$ (56\% of predicted), $\mathrm{FEV}_{1} / \mathrm{FVC}$ (forced vital capacity) of $52 \%$, residual volume (RV) was $2.67 \mathrm{~L}$ (129\%), oxygen saturation level of hemoglobin $\left(\mathrm{SatHbO}_{2}\right)$ was $96 \%$ and chest X-ray showed mild emphysema. A diagnosis was

The work originated from the Department of Cardiologic, Thoracic and Vascular Sciences, University of Padova, Via Giustiniani 2, 35128 Padova, and has been supported by a grant from Takeda Italia SpA.

* The author received a consultancy fee by Takeda Italia SpA for clinical data collection.

Editorial assistance was provided by Selene Mogavero, PhD and Colin G Egan, PhD (Primula Multimedia SrL) and was sponsored by Takeda Italia SpA. 
made of "chronic obstructive pulmonary disease (COPD) in subject professionally exposed to welding fumes" and inhalation therapy with budesonide $1.6 \mathrm{mg}$ plus salmeterol $0.2 \mathrm{mg}$ per day was prescribed, in addition to salbutamol $200 \mu \mathrm{g}$ when needed.

In a follow-up visit (March 2000), during a COPD exacerbation episode, the patient presented with a worsened clinical and functional profile, with exacerbation of dyspnea at rest and on exertion (mMRC scale grade 2) and with copious yellowish sputum. Post BD spirometry showed: $\mathrm{FEV}_{1} 1.85 \mathrm{~L}$ (47.8\%), FEV1/FVC 57\%, RV 3.57 L (165.9\%), carbon monoxide diffusing capacity (DLCO) 28.9, $\mathrm{SatHbO}_{2}$ 96\%. The chest X-ray showed a slightly increased bronchovascular marking at the apex of the heart zone. Therefore, in addition to inhaled therapy, oral antibiotic therapy and prednisone $25 \mathrm{mg}$ was recommended for 15 days, with a decreasing dosage schedule.

Between 2001 and 2006, the patient had on average 2 yearly COPD exacerbations of medium severity, requiring the administra- tion of steroid therapy and oral antibiotics. During a check-up visit in June 2007, the patient showed dyspnea on exertion (mMRC scale grade 1), wheezing and yellowish morning sputum. Post BD spirometry showed: $\mathrm{FEV}_{1} 1.78 \mathrm{~L}$ (49.3\%), $\mathrm{FEV}_{1} / \mathrm{FVC}$ 54.4\%, RV 3.15 L (132.3\%), DLCO 23.8 mmHg min $^{-1} \mathrm{~mm}^{-1}$, SatHbO 2 95\%, pH 7.38, carbon dioxide partial pressure in arterial blood $\left(\mathrm{PaCO}_{2}\right) 69.1 \mathrm{mmHg}$ and partial pressure of oxygen in arterial blood $\left(\mathrm{PaO}_{2}\right) 50$ $\mathrm{mmHg}$. The 6-minute walking test (6MWT) was normal (walked $450 \mathrm{~m}$, no desaturation after exercise). Chest computerized tomography (CT) scan without contrast medium showed signs of bullous emphysema at the level of the right lower lung lobe. The patient was therefore prescribed with inhalation therapy with fluticasone $2 \mathrm{mg}$ and salmeterol $0.2 \mathrm{mg}$ daily, tiotropium bromide $18 \mu \mathrm{g} /$ day and salbutamol $200 \mu \mathrm{g}$ when needed.

Between 2008 and 2011, the patient has been seen in our Department approximately every six months, reporting a progressive de-

Table 1. - Functional parameters pre and post therapy with roflumilast

\begin{tabular}{|c|c|c|c|}
\hline Date & $\begin{array}{c}\text { FEV }_{1} \text { post BD } \\
(\mathrm{L} ; \% \text { of predicted })\end{array}$ & $\mathrm{FEV}_{1} / \mathrm{FVC}(\%)$ & $\begin{array}{cc}\text { RV } & \text { DLCO } \\
(\mathrm{L} ; \% \text { of predicted }) & \left(\mathrm{mmHg} \mathrm{min}^{-1} \mathbf{m m}^{-1}\right)\end{array}$ \\
\hline
\end{tabular}

$\begin{array}{lllll}08.11 .2011 & 1.45 ; 42 & 47.4 & 3.00 ; 120 & 20.8 \\ \text { Before treatment } & & \end{array}$

\begin{tabular}{|c|c|c|c|c|}
\hline $\begin{array}{l}31.01 .2012 \\
\text { Treatment start }\end{array}$ & $1.41 ; 41$ & 45.5 & $3.30 ; 125$ & 20.5 \\
\hline $\begin{array}{l}12.04 .2012 \\
3 \text { months of treatment }\end{array}$ & $1.61 ; 47$ & 54.6 & $3.23 ; 122$ & 25.1 \\
\hline $\begin{array}{l}25.06 .2012 \\
6 \text { months of treatment }\end{array}$ & $1.38 ; 40$ & 45.3 & $3.26 ; 123$ & 29.4 \\
\hline $\begin{array}{l}20.12 .2012 \\
12 \text { months of treatment }\end{array}$ & $1.37 ; 40$ & 49.3 & $3.24 ; 121$ & 30.2 \\
\hline
\end{tabular}

$\mathbf{F E V}_{\mathbf{1}}$, forced expiratory volume in the first second; FVC, forced vital capacity; BD, bronchodilator test; $\mathbf{R V}$, residual volume; DLCO, carbon monoxide diffusing capacity. 
A

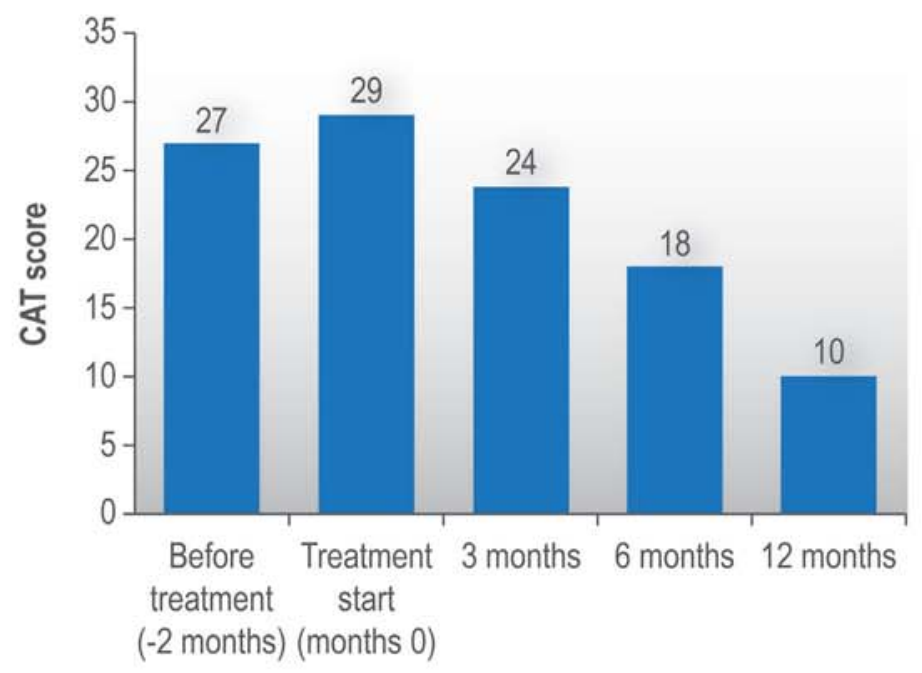

B

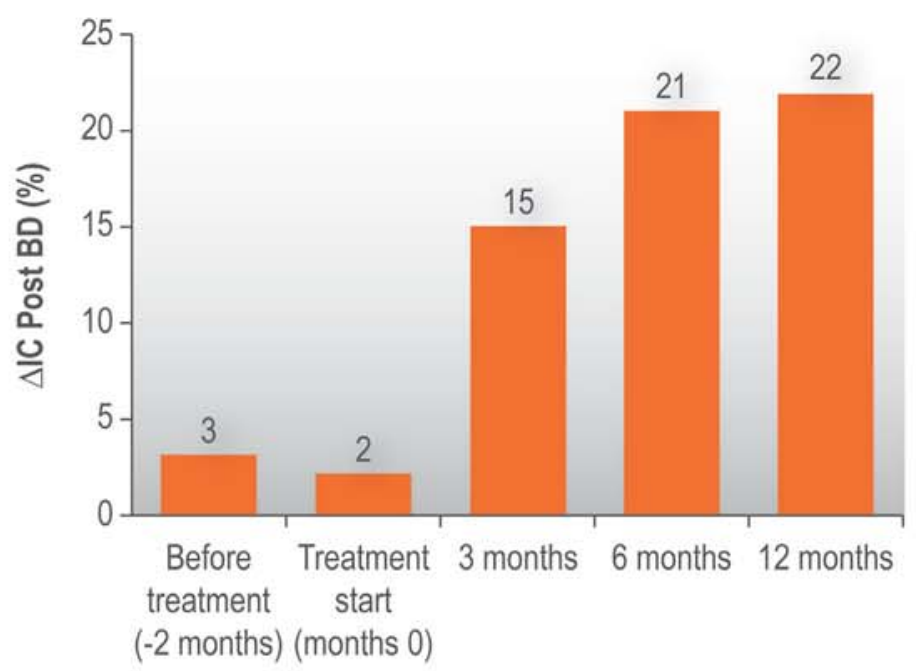

c

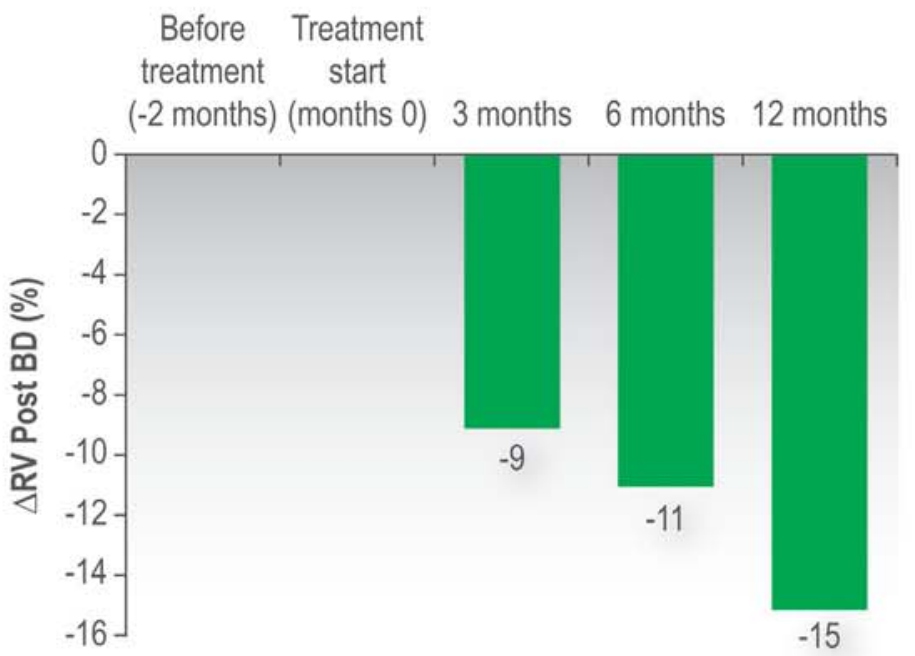

Fig. 1. - Improvements in patient's conditions after treatment with roflumilast, as depicted by A) CAT scores; B) IC post $\mathrm{BD}$ variations and C) RV post $\mathrm{BD}$ variations. CAT, COPD assessment test; IC, inspiratory capacity; BD, bronchodilator test; $\mathbf{R V}$, residual volume. 
terioration of morning sputum and dyspnea on exertion (mMRC grade 2). COPD exacerbations requiring steroid therapy and oral antibiotics became more frequent (on average 3 per year). Arterial blood gas analysis showed resting hypoxemia (mean value of 76.5 $\mathrm{mmHg}$ ) and hypercapnia (mean value of $45.6 \mathrm{mmHg}$ ).

In November 2011, during a check-up visit, the patient complained about a marked exertional dyspnea (mMRC scale grade 3) and had recently recovered from a moderate COPD exacerbation. The functional profile (table 1 and figure 1, before treatment data) was unchanged, as well as the radiological one (by means of a CT scan without contrast medium performed in January 2012). $\mathrm{SatHbO}_{2}$ was 94.7\%, pH 7.382, $\mathrm{PaO}_{2} 77.3$ and $\mathrm{PaCO}_{2}$ 47.2. The 6MWT showed desaturation after exercise (from $95 \%$ to $93 \%$ ) after walking $420 \mathrm{~m}$. Therapy was unchanged.

The $31^{\text {st }}$ of January 2012 (treatment start) the patient was checked again (table 1 and figure 1) and, meeting the eligibility criteria for roflumilast therapy (as determined by Italian Drug Agency), he was prescribed this medication $(500 \mu \mathrm{g} /$ day $)$. After 10 days of treatment the patient complained about profuse diarrhea for three days, therefore it was agreed to suspend the drug for 10 days and, in case of remission of diarrhea, to resume therapy. It must however be noted that, in the same days, the patient had taken a short course of oral amoxicillin for dental treatment.

The patient followed directions and restarted therapy with roflumilast, without subsequently reporting of any specific side effects or weight loss. Since then and until December 2012 (after 12 months of roflumilast treatment) he has not had any COPD exacerbations nor had he to take steroid therapy or oral antibiotics. Quality of life improved as evidenced by the COPD assessment test (CAT - from 27 prior to treatment with roflumilast to 10 , after 12 months of treatment, figure $1 \mathrm{~A}$ ), reducing dyspnea
(mMRC scale grade 0-1) and productive cough. Lung function tests highlighted an increase of inspiratory capacity (IC) after bronchodilator, with response already after 3 months of therapy (15\% increase) and further increase at the last control $(+22 \%)$ (figure 1B). At the same time, a decrease in RV after bronchodilator was noted, made more apparent in the last control (15\% reduction) (figure 1C). The diffusion capacity of lung gas, although not particularly compromised during all the examined years, presented a progressive increase, reaching $+47 \%$. at the last control (after 12 months of treatment, table 1). In December 2012, the 6WMT was normal (walked for $490 \mathrm{~m}$ with no desaturation after exercise).

\section{Discussion}

The clinical case presented here describes the history of a patient with COPD stage D according to Guidelines GOLD 2013 [1], in a subject professionally exposed to welding fumes. The course of the disease has been long and characterized by a progressive deterioration. The therapeutic option of adding roflumilast $(500 \mu \mathrm{g} / \mathrm{day})$ to the triple combination therapy, in line with the recommendations of the "Global Initiative for Chronic Obstructive Lung Disease" (GOLD) Guidelines [1, 2], has been successful. It allowed a rapid clinical and functional improvement of the patient, and a good therapeutic control of the disease.

Efficacy of roflumilast treatment was further evidenced by the dramatic reduction of exacerbations and a marked improvement of respiratory symptoms, also evidenced by the improvement in CAT test scores. This score is particularly meaningful since it allows us to compare it to those reported in a recent meta-analysis [3], in which roflumilast led to an improvement in respiratory symptoms and quality of life (analyzed by the St George's Respiratory Questionnaire), al- 
though was not found to be statistically significant.

Roflumilast therapy also led to an improved tolerance to exercise, as evidenced by the positive outcomes of the $\mathrm{SatHbO}_{2}$ test, by the distance walked during the 6MWT and by the dyspnea levels after exercise (mMRC scale). This is in line with what has been previously documented by O'Donnell et al. [4] who showed an improvement in oxygen saturation and TDI (Transition Dyspnea Index) during cycling exercise in COPD patients treated with roflumilast compared to those treated with placebo. The same authors did not document any particular change in hyperinsufflation in static conditions (as well as during exercise) in patients treated with roflumilast. Our patient, instead, did experience a progressive reduction of the RV and an increase in IC after bronchodilator test.

In contrast to what has been reported by other authors $[3,5,6]$, in our patient no improvement in $\mathrm{FEV}_{1}$ after bronchodilator test has been observed with roflumilast. On the other hand, a significant increase in lung gas diffusion capacity has been documented, which suggests a decreased ventilation-perfusion heterogeneity, although the mechanism of these changes is still unclear.

Treatment with roflumilast was well tolerated and safe, apart from the onset of diarrhea, which was documented in the course of concomitant antibiotic therapy, as already shown in other studies $[3,7]$. The patient agreed to temporarily suspend roflumilast and then resume it and this regimen has proven to be efficient and may be suggested in common clinical practice.

\section{Riassunto}

Viene di seguito presentato un caso clinico di un uomo di 72 anni, con una lunga storia di bronco pneumopatia cronica ostruttiva (BPCO, paziente D secondo le Linee Guida
GOLD 2013) in soggetto professionalmente esposto a fumi di saldatura. La diagnosi si basava sui valori spirometrici e l'esame a raggi X del torace. Fin dal 1997, il paziente è stato trattato con diverse terapie tradizionali, in particolare cortisonici e broncodilatatori per via inalatoria ad alte dosi, con scarso controllo clinico e frequenti riacutizzazioni bronchitiche. Nel gennaio 2012 si iniziava il trattamento con roflumilast 500 $\mu \mathrm{g}$ una volta al giorno e da allora le condizioni del paziente, intese come sintomi respiratori, numero di esacerbazioni e valori spirometrici, sono gradualmente migliorate. Roflumilast è risultato altamente efficace in questo caso di grave BPCO difficile da trattare.

Parole chiave: Roflumilast, BPCO, Caso clinico.

\section{References}

1. Global Initiative for Chronic Obstructive Lung Disease (GOLD). Global Strategy for the Diagnosis, Management and Prevention of COPD. 2013. Available at www.goldcopd.org.

2. Osthoff M, Jenkins C, Leuppi JD. Chronic obstructive pulmonary disease - a treatable disease. Swiss Med Wkly 2013; 143.

3. Yan J-H, Gu W-J, Pan L. Efficacy and safety of roflumilast in patients with stable chronic obstructive pulmonary disease: A meta-analysis. Pulm Pharmacol Ther 2013; 27: 83-9.

4. O'Donnell DE, Bredenbröker D, Brose M, Webb KA. Physiological effects of roflumilast at rest and during exercise in COPD. Eur Respir J 2012; 39: 1104-1112.

5. Calverley PMA, Rabe KF, Goehring U-M, et al. Roflumilast in symptomatic chronic obstructive pulmonary disease: two randomised clinical trials. Lancet 2009; 374 (9691): 685-694.

6. Fabbri LM, Calverley PMA, Izquierdo-Alonso $\mathrm{JL}$, et al. Roflumilast in moderate-to-severe chronic obstructive pulmonary disease treated with longacting bronchodilators: two randomised clinical trials. Lancet 2009; 374 (9691): 695-703.

7. Rabe KF. Update on roflumilast, a phosphodiesterase 4 inhibitor for the treatment of chronic obstructive pulmonary disease. Br J Pharmacol 2011; 163: 53-67. 


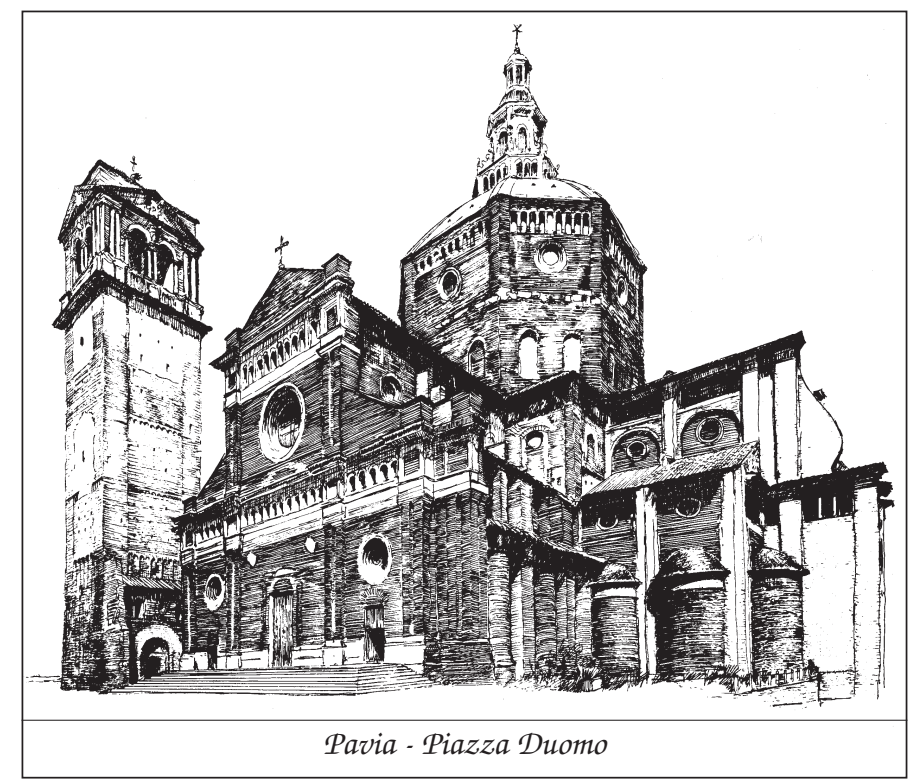

